\title{
Face recognition method based on GA-BP neural network algorithm
}

https://doi.org/10.1515/phys-2018-0126

Received October 2, 2018; accepted November 14, 2018

\begin{abstract}
In order to recognize faces, face recognition methods need to be studied. When a face is identified by the current method, the image denoising effect is poor, the face image recognition result thus has error, the time used to recognize the face image is long, the signal to noise ratio, the recognition result and the recognition efficiency are low. Based on the GA-BP neural network algorithm, a face recognition method is proposed. A mixed denoising model of face images is constructed by combining dictionary based sparse representation with non-local similarity. The principal component analysis method is used to extract the feature of the face image after denoising and staining the eigenvector of the face image. The GA$\mathrm{BP}$ neural network algorithm is used to optimize the initial weights and thresholds so as to achieve the optimal value. The feature vectors of face images are ted into the genetic neural network to complete face recognition. Experimental results show that the proposed method has high signalto-noise ratio, accuracy and recognition efficiency.
\end{abstract}

Keywords: GA-BP neural network, face recognition, image denoising, feature extraction

PACS: 07.05.Pj, 07.05.Kf, 07.05.Mh

\section{Introduction}

With the development of high-tech technology and the occurrence of terrorist incidents, people's privacy and security issues have increasingly become the focus of attention. Identity identification and authentication exist in the fields of national security, public security, judicial, elec-

\footnotetext{
*Corresponding Author: Jing Liu: School of Mathematics and Statistics, Nanyang Institute of Technology, Nanyang, 473000 China, E-mail: Jingliu1211@126.com

Muhammad Aqeel Ashraf: International Water, Air Soil Conservation Society INWASCON, 59200, Kuala Lumpur, Malaysia,

E-mail: ashraf@inwascon.org.my
}

tronic commerce, e-government, security inspection, security monitoring and so on [1]. Traditional authentication methods are easy to lose, forge, damage, forget, be stolen by others as well as other drawbacks, which canot meet the need of communities. Especially after the 9.11 incident in the United States, the national security problem has been severely tested. The guarantee of information security by an effective means of authentication is an urgent problem waiting to be solved by the government and the scientific community at present [2].

Face recognition is an identification method based on human facial features. It has many advantages such as initiative, non-aggression and user-friendliness. In particular, face recognition is superior to iris, fingerprints, and gait recognition methods [3]. Face recognition is the recognition of the input face image or video. First, it determines whether there is a face. If there is a face, the position, size and other information of each face are given, and the identity features of each face are extracted and compared with the face in the database to realize identification [4]. Face recognition involves many research fields, such as image processing, machine vision and pattern recognition. The research results have broad application prospects, which can be used in judicial authentication, civil aviation security inspection, customs authentication, intelligent video surveillance, and the verification of various bank cards and financial card holders. However, because of the complexity of face change, it is very difficult to express and extract features, which makes face recognition a very challenging task, and it is necessary to study face recognition methods [5].

Yuan Heng, Wang Zhihong and Jiang Wentao proposed a 3D face recognition method based on singular point neighborhood structure. In this method, the target region was segmented on the face texture image, the feature sub-regions were divided, and the neighborhood structures of two-dimensional singular points and singular points were extracted. The 3D singular points and their neighborhood structures were marked on the geometric information of face space, and the 3D information of the singular point and its neighborhood structures was used to represent the face features. This method used the near- 
est point method of singular point neighborhood structure to identify face identity information. This method ignored a variety of noise signals in the face image, and the recognition results were inaccurate and the accuracy of recognition results was low [6]. Sun Jinguang and Meng Fanyu proposed a feature weighted fusion face recognition method based on deep neural network. This method used active shape model to extract the main feature points of the face, and sampled different parts of the face according to the main feature points. The obtained sampling blocks were input into the corresponding deep belief network respectively to train and obtain the best network parameters. The similarity vectors of each region were obtained by using Softmax regression, and the comprehensive similarity score was obtained by weighting and fusing the similarity vectors of multiple regions. According to the result, the face recognition was completed. There were many algorithms involved in this method. In the calculation process, the error was easy to appear, and there was a problem of low accuracy of recognition results [7]. Li Yaqian, Zhang Shaowei, Li Haibin, et.al proposed a face recognition method based on Gabor wavelet and mutual covariance reduction operation, to extract Gabor features of face image. Aeighted mutual covariance matrix was used to reduce the dimension and feature extraction of processed feature images. Through the nearest neighbor classifier, the face recognition was completed according to the classification results. The recognition process of this method was tedious, the recognition took a long time, and there was a problem of low recognition efficiency [8]. Lin Yunfeng and Zhang Lihua proposed a face recognition method based on greedy approximate algorithm. The main idea of this method was that the face image of album structure reflected the compatibility between tags, and the number of tags in the album could be controlled by tag cost. According to the tag cost, the personal information in the album was modeled, and the greedy approximation algorithm to solve the NP problem in the modeling process was given. The model parameters were obtained by SSVM training, and face recognition was completed by the maximum membership degree principle. This method could not effectively remove many noises in the face image, and the image denoising was low after de-noising [9].

To sum up, a face recognition method based on GA-BP neural network algorithm is proposed. The specific steps are as follows:

A mixed denoising model of face images is constructed by sparse representation and non-local similarity.

Principal component analysis (PCA) is used to extract features in face images.
GA-BP neural network algorithm is used to complete face recognition.

The experimental results and analysis. Section shows that the algorithm based on GA-BP neural network is effective in three aspects: signal-to-noise ratio, recognition accuracy and recognition efficiency. Results and discussion.

In the section, the focus of the next step is put forward.

\section{Methods}

\subsection{Mixed denoising model}

The dictionary based image sparsity is used to construct a denoising variational model, and a weight factor is designed to suppress the interference of the impact noise in the data fidelity term in the model. The initial denoising of mixed noise images is achieved through the idea of nonlocal mean. In the obtained image, the masking matrix is constructed to exclude the impact noise points and obtain non-local similarity prior knowledge. The non-local similarity prior and sparse prior are fused into the regular terms of the variational model, and a mixed denoising model is constructed to obtain the denoised face image.

The general accuracy of input face images is high and there is a lot of redundant information. In order to avoid the complex structure of genetic neural network, image compression must be performed before the genetic neural network is input [10]. Let $f(x)$ be used for interpolation and $h(x)$ for interpolation kernel, then the interpolation Eq. is:

$$
f(x)=\sum_{i=0}^{K-1} C_{i} h\left(x-x_{i}\right)
$$

where, $C_{i}$ represents the weight coefficient. The face recognition method based on the GA-BP neural network algorithm uses cubic function, and the size of the interpolated neighborhood is $4 \times 4$. Thus, the value of the output pixel value is the weighted average of the effective points contained in the $4 \times 4$ matrix.

$x$ represents the compressed face image; $x_{i, j}$ represents the pixel location located at the position $(i, j) ; y$ represents the image after the face image $x$ is contaminated by noise, and the expression of the mixed noise model is as follows:

$$
y_{i, j}=\left\{\begin{array}{l}
d_{\min }, \text { probabilityiss } / 2 \\
d_{\max }, \text { probabilityiss } / 2 \\
d_{i, j}, \text { probabilityisr }(1-s) \\
x_{i, j}+v_{i, j}, \text { probabilityis }(1-r)(1-s)
\end{array}\right.
$$

where, $v_{i, j}$ is the Gauss noise value which obeys the zero mean distribution and the variance is $\sigma$. 
Set $\left[d_{\min }, d_{\max }\right]$ be the range of the gray value of the noise image $y$. When the face image is contaminated with salt and pepper noise, $s(0 \leq s \leq 1)$ is the ratio of the noise of salt and pepper. The value of the noise point is only two extreme cases: $d_{\min }$ or $d_{\max }$, and their probability is $s / 2$. For random impulse noise, the noise ratio is $r(0 \leq r \leq 1)$, the noise point value is $d_{i, j}\left(d_{\min }<d_{i, j}<d_{\max }\right)$, and the probability of $y_{i, j}=d_{i, j}$ is $r(1-s)$. The pixel value that is only contaminated by Gauss noise is $y_{i, j}=x_{i, j}+v_{i, j}$ and the probability is $(1-r)(1-s)$.

Let $x_{i} \in R_{i}$ represent an image neighborhood block with the size of $\sqrt{n} \times \sqrt{n}$ and $R_{i}$ be a matrix vector. Through it, one image block $x_{i}$ in image $x$ can be removed. According to the theory of sparse representation, dictionary $\Phi=\left[\Phi_{1}, \Phi_{2}, \cdots, \Phi_{n}\right]$ can be used to make sparse coding of $x_{i}$, then $x_{i}$ can be expressed as:

$$
x_{i}=\Phi \cdot \alpha_{i},
$$

where, $\alpha_{i}$ is a sparse coding vector. The encoded image $x$ can be expressed as:

$$
x=\Phi \cdot \alpha
$$

where, $\alpha$ represents a sparse coding vector. The choice of dictionary has a great influence on the sparse encoding and reconstruction of signals [11]. In the GA-BP neural network algorithm, the dictionary must be learned in advance through uncontaminated natural face images. By learning from natural face images, a PAC dictionary is used for sparse representation. PCA dictionary is self-adaptive. Principal components are extracted by principal component analysis (PCA) to update the atoms in the dictionary [12]. For image $y$ contaminated by Gauss noise, coding model $\alpha_{\text {new }}$ can be expressed as:

$$
\alpha_{\text {new }}=\underset{\alpha}{\arg \min }\|y-\Phi \cdot \alpha\|_{2}^{2}+R(\alpha),
$$

where, $R(\alpha)$ is a regular term, which is to describe some properties of the solution to limit the solution space, so that the solution of the problem has favorite properties and thus a stable solution is obtained. According to the deterministic regularized theory, the maximum posterior solution of the coding vector obtained by Gauss noise is obtained [13]. For the face images contaminated by mixed noise, the distribution of noise is very different from that of Gauss noise. Under the influence of impact noise, the residual error $y-\Phi \alpha$ of data fitting for mixed noise is more irregular than that of Gauss noise. Therefore, in Eq. (5), $l_{2}$ norm is used to represent that the data fitting residuals is not applicable to the restoration of mixed noise contaminated images. If the data fidelity term is changed to make the distribution of the residual difference more similar to the distribution of Gauss noise, the $l_{2}$ norm can still be used to represent the coded residual under mixed noise, making the removal of the mixed noise easier to handle. By weighting data fidelity items, the distribution is more regular. So, let:

$$
e=\left[e_{1}, e_{2}, \cdots, e_{N}\right]=y-\Phi \alpha,
$$

where, the residual $e_{i}=y-\Phi \alpha . \min \sum_{i=1}^{N} f\left(e_{i}\right)$ is used to replace $e_{i}$. Function $f$ controls the distribution of every residual, so that the residual distribution is more consistent with the residual distribution under Gauss noise. $f$ should meet the following nature:

$$
\begin{gathered}
f(e) \geq 0 \text { and } f(0)=0 \\
f\left(e_{i}\right) \geq f\left(e_{j}\right),\left|e_{i}\right|>\left|e_{j}\right| \\
f(e)=f(-e)
\end{gathered}
$$

The residual distribution in mixed noise will have heavy tailed distribution. The heavy-tailed distribution is similar to the normal probability distribution, but its tail is thicker than the tail of the normal distribution, with the characteristics of peak and heavy-tailed [14]. For each residual error, the weighted residuals are:

$$
e_{i}^{w}=w_{i}^{1 / 2} e_{i},
$$

where, $w_{i}$ is a diagonal element. In the mixed denoising, the residual can be divided into two categories: for the noise contaminated by Gauss noise, the residual error basically obeys the Gauss distribution and can remain unchanged, that is, the weight is approximately 1; Residues at impact noise points should be weighted to reduce the heavy tail distribution [15]. From the above analysis, $f\left(e_{i}\right)$ can be set to $f\left(e_{i}\right)=\left(w_{i}^{1 / 2} e_{i}\right)^{2}$, and the improved mixed noise denoising model is:

$$
\alpha_{\text {new }}=\underset{\alpha}{\arg \min }\left\|w^{1-2}(y-\Phi \alpha)\right\|_{2}^{2}+R(\alpha),
$$

where, $w$ is diagonal weighting matrix and diagonal element $w_{i i}=w_{i}$. The pixels contaminated by impact noise should have a smaller weight, which can reduce the influence of impact noise on coding. For pixels that are not contaminated by impact noise, the weight value should be close to 1 . Because in the sparse representation of the impact noise points, there will be large coding residuals, which is set according to the encoding residual $e_{i}$ to the weight $w_{i i}$, and the size of $w_{i i}$ and $e_{i}$ is inverse proportional. Set $w_{i i} \in[0,1]$, the calculation formula of $w_{i i}$ is as follows:

$$
w_{i i}=\exp \left(-b e_{i}^{2}\right)
$$


where, $b$ is a normal number, which can control the decay rate of $w_{i i}$. In order to make the denoising model (9) more effective for mixed noise removal, it is necessary to apply prior knowledge of the face image to the design of regular term $R(\alpha)$. The two prior knowledge of local sparsity and non local self-similarity are fused into a regular term of a variational model, and a more accurate sparse representation coefficient will be obtained for the construction of the denoising model.

When the face image contains the mixed noise composed of Gauss noise and impact noise, it can cause interference to the non local similarity solution in the face image [16]. The face recognition method based on GA-BP neural network firstly preprocesses the noise image $y$, and preliminarily removes the impulse noise in the face image. Using fuzzy weight non local average algorithm, the initial image $x^{(0)}$ can be obtained by filtering the noise image. The fuzzy weight non local average algorithm uses the non local similarity of the face image, and the noise of the face image is removed by obtaining the fuzzy weighted non local mean value of the pixel points. It is more accurate to seek nonlocal similarity prior knowledge through $x^{(0)}$. The matrix $z$ is constructed according to the initial image $x^{(0)}$ :

$$
z=\left\|y-x^{(0)}\right\|
$$

A threshold value of $2 \sigma$ is set to judge whether the pixel at the position $(i, j)$ in noise image $y$ is the impact noise point. If $z(i, j) \geq 2 \sigma$, it is determined that the pixel is the impact noise point, otherwise it is the Gauss noise point. An identification matrix $d(i, j)$ is constructed by the matrix $z$ :

$$
d(i, j)= \begin{cases}1, & z(i, j)<2 \sigma \\ 0, & z(i, j) \geq 2 \sigma\end{cases}
$$

When solving the non local similar priori of face image $x^{(0)}$, matrix $d(i, j)$ is used as a mask matrix, and a new image matrix $x^{(1)}$ is preliminarily excluded from the impact noise point in $x^{(0)}$, that is, the non local similarity prior to the face image in Gauss noise environment is obtained only. The result will be closer to the true information of the face image [17]. For the image block $x_{i}$ in $x^{(1)}$, a similar block is looked for in the large enough window area centered on $i$. If the Euclidean distance between image blocks $x_{i}^{q}$ and $x_{i}$ does not exceed a predetermined threshold, then they are considered to be similar. If there are $L$ image blocks similar to $x_{i}$, the weighted average of them can be obtained as following.

$$
x_{i}=\sum_{q=1}^{L} b_{i}^{q} x_{i}^{q},
$$

where, $q$ represents the number of image blocks. $x_{i}$ can be used to estimate image block, and the distance between weights $b_{i}^{q}$ and $x_{i}$ is inversely proportional to $x_{i}^{q}$.

$$
b_{i}^{q}=\exp \left(-\left\|x_{i}-x_{i}^{q}\right\|_{2}^{2} / h\right) / \omega,
$$

where, $h$ is a pre-set scalar, and $\omega$ is normalization factor. If the advanced learning dictionary $\Phi$ is used to encode the image block and its non local similar block, then $x_{i}=\Phi \alpha_{i}$, and the non local similar block $x_{i}=\Phi \alpha_{i}$, the coding coefficients $\alpha_{i}$ and $\alpha_{i}$ are similar. The face recognition method based on GA-BP neural network algorithm takes $\sum_{i}\left\|\alpha_{i}-\mu_{i}\right\|$ as a regular term to substitute into Eq. (9), then obtaining.

$$
\alpha_{n e w}=\underset{\alpha}{\arg \min }\left\|w^{1 / 2}(y-\Phi \alpha)\right\|_{2}^{2}+\|\alpha-\mu\|_{1},
$$

where, represents the coding coefficient. In the above model, the data fidelity term is weighted. The regular term combines the sparse prior and the non local similar priori, which makes the denoising model integrate more prior knowledge, and the denoising image will be closer to the real life image [18].

Under the mixed noise composed of Gaussian noise and impulse noise, the initial value of coding residue $e$ can be set to $e^{(0)}$ by the initial image $e^{(0)}$, and the calculation formula of $e^{(0)}$ is as follows:

$$
e^{(0)}=y-x^{(0)}
$$

The weighting matrix $w$ is initialized by $e^{(0)}$ through Eq. (10).

When $w$ is determined, the algorithm model (15) becomes a sparse coding problem of $l_{1}$ norm. Face recognition algorithm based on GA-BP neural network algorithm can solve the model by iterative reweighting algorithm. Let $V$ be a diagonal matrix and initialize it as a unit matrix. At the $k+1$ th iteration, the element in $V$ is updated to:

$$
V_{i i}^{(k+1)}=\mu /\left(\left(\alpha_{i}^{(k)}-\mu_{i}\right)^{2}+\varepsilon^{2}\right)^{1 / 2},
$$

where, $\varepsilon$ is scalar, and the mixed denoising model $\alpha^{(k+1)}$ is obtained after update. The expression of $\alpha^{(k+1)}$ is as follows:

$$
\alpha^{(k+1)}=\left(\Phi^{T} w \Phi+V_{i i}^{(k+1)}\right)^{-1}\left(\Phi^{T} w y-\Phi^{T} w \Phi \mu\right)+\mu
$$

\subsection{Feature extraction of face Image}

The face recognition method based on GA-BP neural network algorithm is used to extract features from face images by principal component analysis. The principal component analysis method is the statistical analysis method 
based on K-L transformation. The essence of principal component analysis is the projection of the high dimensional space sample data into the low dimensional space by linear transformation while retaining the original data as much as possible in the original high dimensional vector can be obtained by rebuilding the low dimension vector and the eigenvector restructure [19]. Principal component analysis (PCA) obtains the orthogonal basis of high dimensional face image space by linear transformation to form feature space. For denoising two-dimensional $n \times m$ image $R$, face image training set $\left\{R_{i} \mid i=1,2, \cdots, k\right\}, k$ is the total number of training samples, and the image mean is $\bar{R}$.

$Z_{i}$ is the standard training sample for face images. The expression is as follows:

$$
Z_{i}=R_{i}-\bar{R}
$$

The covariance matrix $\operatorname{Cov}$ is obtained by the standard training sample $Z_{i}$.

$$
\operatorname{Cov}=Z Z^{T}
$$

Let matrix $A$ be a $n \times \iota$ matrix with rank $\iota$, there are two orthogonal matrices $U$ and $V$, and diagonal matrix $\Lambda$, so that matrix $A$ satisfies the following equations:

$$
A=U \Lambda^{\frac{1}{2}} V^{T}
$$

$\left\{\lambda_{i} \mid i=0,1, \cdots, \iota-1\right\}$ is set as the non zero eigenvalues of matrices $A A^{T}$ and $A^{T} A, u_{i}$ and $v_{i}$ are the eigenvectors of $A A^{T}$ and $A^{T} A$ corresponding to $\lambda_{i}$, respectively. According to the singular value decomposition theorem, $\left\{\lambda_{i} \mid i=0,1 . \cdots, \iota-1\right\}$ is the $\iota$ non zero eigenvalues of covariance matrix $\operatorname{Cov}, u_{i}$ and $v_{i}$ are the eigenvectors of $\lambda_{i}$, and the orthogonal normalization vector of the face image is obtained.

$$
u_{i}=\frac{1}{\sqrt{\lambda_{i}}} Z v_{i} \varsigma,
$$

where, $\varsigma$ represents the sensitive factor and the value is taken in the interval $[0,10]$. The higher the sensitivity factor is, the more accurate the feature vector is.

\subsection{Face recognition based on GA-BP neural network algorithm}

BP network is a multilayer feedforward network trained by the error back propagation algorithm. Its structure is similar to the human brain, and is composed of a large number of neurons connected to each other, which has a highly nonlinear mapping ability, and can carry out complex nonlinear mapping. Face recognition can use the feature vector of the face image as input, which kind of person is the problem of mapping the complex function of output, so it can be used for face recognition by neural network [20-26].

$\mathrm{BP}$ network algorithm is slow to learn and easy to fall into local optimum. In order to overcome this shortcoming, a face recognition method based on GA-BP neural network algorithm is proposed. First, a denoising model is constructed to remove the noise in face images. The feature vector of the face image is obtained by the principal component analysis, and the genetic algorithm is used to optimize the initial weight and threshold of the neural network so that it can reach the optimal value. Then, the optimal initial weights and thresholds are brought to the GABP neural network for network training. When training is completed, the feature vectors of face images are input into GA-BP neural network to complete face recognition. The flow chart of face recognition method based on GA-BP neural network algorithm is shown in Figure 1.

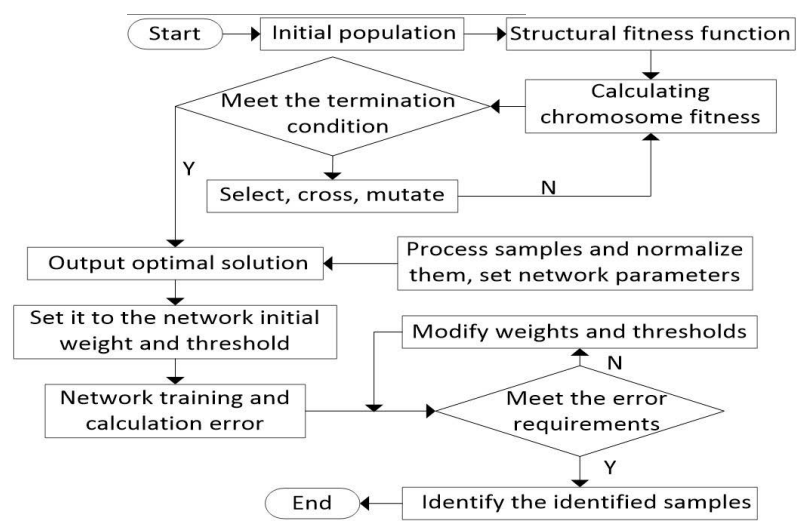

Figure 1: Flow chart of GA-BP neural network algorithm

The main steps of the algorithm are as follows:

Firstly, initialization of the population. Each individual is composed of weights and thresholds of each layer and coded by real number encoding.

Secondly, the fitness function is determined. $y_{k}^{p}$ represents the initial weights of the GA-BP neural network, and $o_{k}^{p}$ represents the initial threshold of the GA-BP neural network. Fitness function $F$ is defined as the reciprocal of the absolute error sum of initial weight $y_{k}^{p}$ and initial threshold $o_{k}^{p}$ in GA-BP neural network. The expression of fitness function $F$ is as follows:

$$
F=\frac{1}{\sum_{p=1}^{N} \sqrt{\sum_{k=1}^{m}\left(y_{k}^{p}-o_{k}^{p}\right)^{2}}}
$$


Thirty, the selection operation is performed. The roulette method is used to select operation, the probability of individual $i$ being selected is $P_{i}$, and the calculation formula of probability $P_{i}$ is as follows:

$$
P_{i}=\frac{F_{i}}{\sum_{i=1}^{c} F_{i}},
$$

where, $c$ is the total number of populations.

Fourthly, a cross operation. This cross operation is carried out by real number cross method. The crossover probability is used to exchange some genes and form two new individuals. The operation method is as follows:

$$
\left\{\begin{array}{l}
g_{k_{1} j}=g_{k_{1} j} a+g_{k_{2} j}(1-a) / P_{i} \\
g_{k_{2} j}=g_{k_{2} j} a+g_{k_{1} j}(1-a) / P_{i}
\end{array},\right.
$$

where, $g_{k_{1} j}$ and $g_{k_{2} j}$ represent $k_{1}$ and $k_{2}$ individuals at the $j$ th gene and $a$ is the random number of $[0,1]$.

The fifth step is the mutation operation. In order to increase population diversity, some genes in the population will mutate with mutation probability. The operation method is as follows:

$$
g_{i j}=\left\{\begin{array}{l}
g_{i j} a_{2}+\left(g_{i j}-g_{\max }\right) a_{1}\left(1-s / s_{\max }\right), a_{2} \geq 0.5 \\
g_{i j} a_{2}+\left(g_{\min }-g_{i j}\right) a_{1}\left(1-s / s_{\max }\right), a_{2}<0.5
\end{array}\right.
$$

where, $g_{\max }$ and $g_{\min }$ represent the upper and lower bounds of gene $g_{i j} ; a_{1}$ is the random number; $s$ is the number of current iterations, $s_{\max }$ is the maximum evolutionary times, and $a_{2}$ is the random number of $[0,1]$.

And six is to set the standard threshold $\vartheta$. When the fitness function value is less than $\vartheta$, it satisfies the end condition of the algorithm, and the optimal weight value $y_{k}^{p}$ and the threshold value $o_{k}^{p}$ are obtained; if it is not satisfied, then return to step three.

Normalization processing favors part of the seventh step. In order to improve the convergence speed, the output of neural network is normalized. The input vector is $x$, the output vector is $x^{\prime}, x_{\max }$ is the maximum of the input vector $x$, and $x_{\min }$ is the minimum value of the input vector $x$, then the input vector standardization process can be described as:

$$
x^{\prime}=\frac{x-x_{\min }}{x_{\max }-x_{\min }}
$$

Finally, the optimal weights $y_{k}^{p}$ and the threshold $o_{k}^{p}$ solved in the step six and the data $x^{\prime}$ processed by the step seven are taken into the GA-BP neural network to make grid training, and get the recognition result $x_{w}$, so as to complete the face recognition.

$$
x_{w}=\int_{x^{\prime}}^{\vartheta} F / u_{i}
$$

\section{Results}

In order to verify the overall effectiveness of the face recognition method based on the GA-BP neural network algorithm, it is necessary to test the face recognition method. The test platform is Matlab and the operating system is Windows 10. Face image contains a lot of noise, including salt and pepper noise, impulse noise and Gaussian noise, which affect the recognition of face image. The face recognition method based on GA-BP neural network algorithm, the 3D face recognition method based on the neighborhood structure of singular point and the feature weighted fusion face recognition method based on the depth neural network are tested to remove the noise signal in the face image (Figure 2), and the test results are shown in Figure 3.

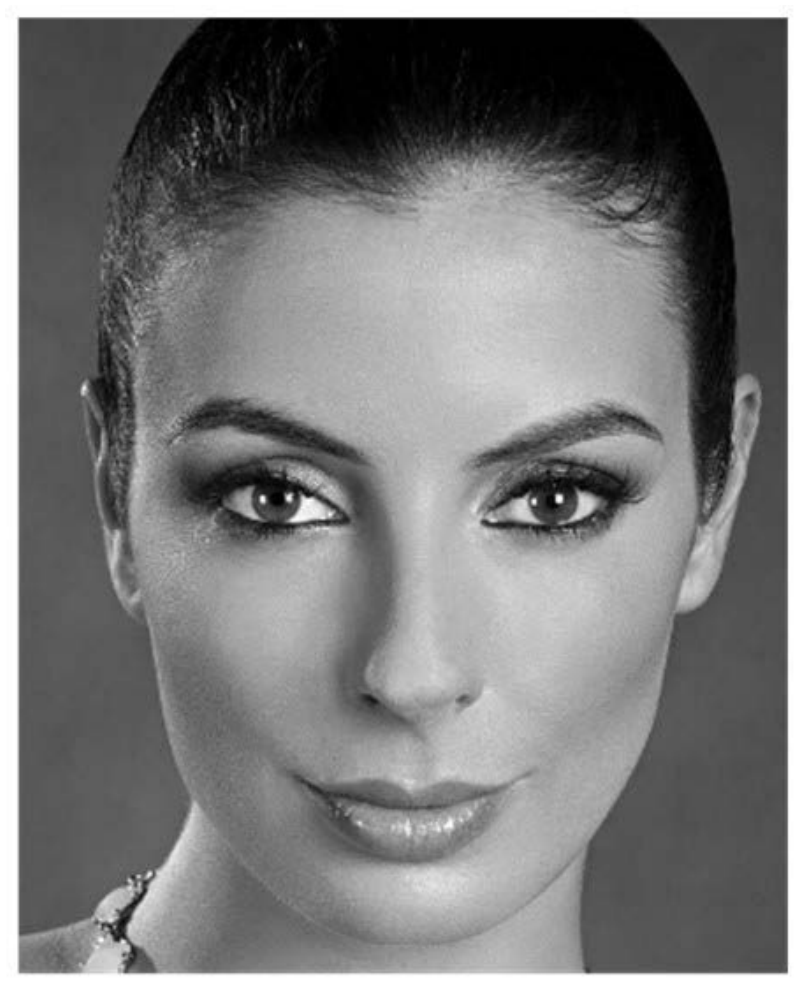

Figure 2: Face image

Figure 3(a) is the signal frequency of the original face image. Figure $3(\mathrm{~b})$ is the signal frequency of the face recognition method based on GA-BP neural network algorithm. In comparison of Figure 3(a) and 3(b), it can be seen that the fluctuation of signal frequency in Figure 3(b) is small. Figure 3(c) and 3(d) are the signal frequency of denoising Figure 2 by using the 3D face recognition method based on singular point neighborhood struc- 


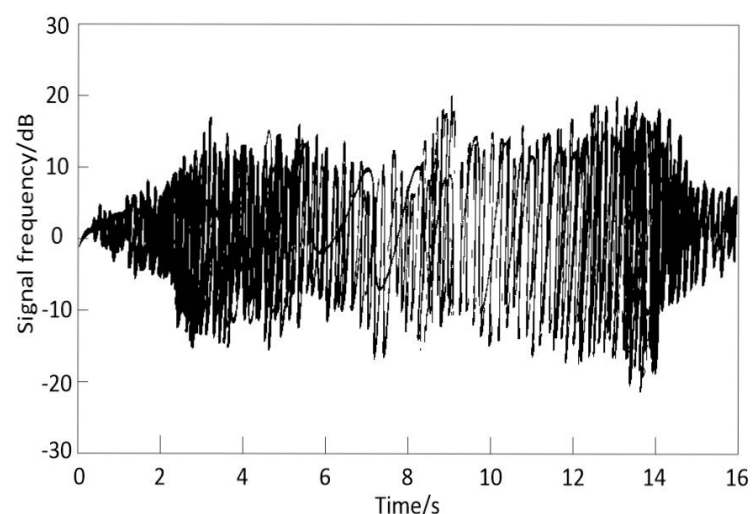

(a) The signal frequency of the original face image

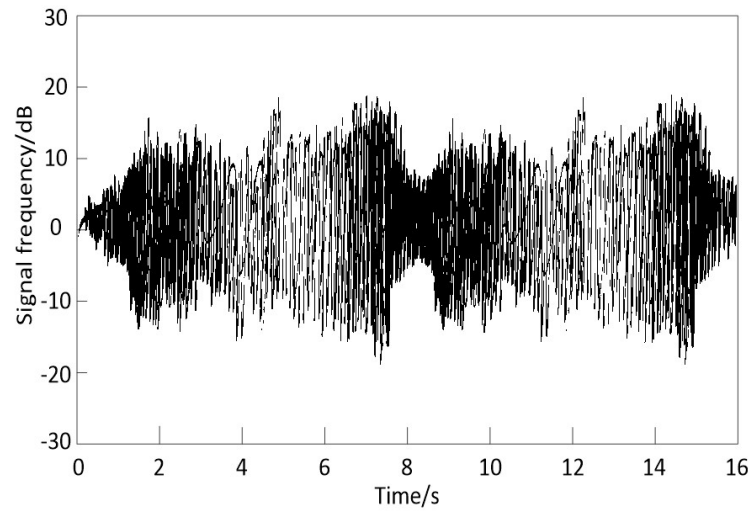

(c) Denoising results of 3D face recognition method based on singular point neighborhood structure

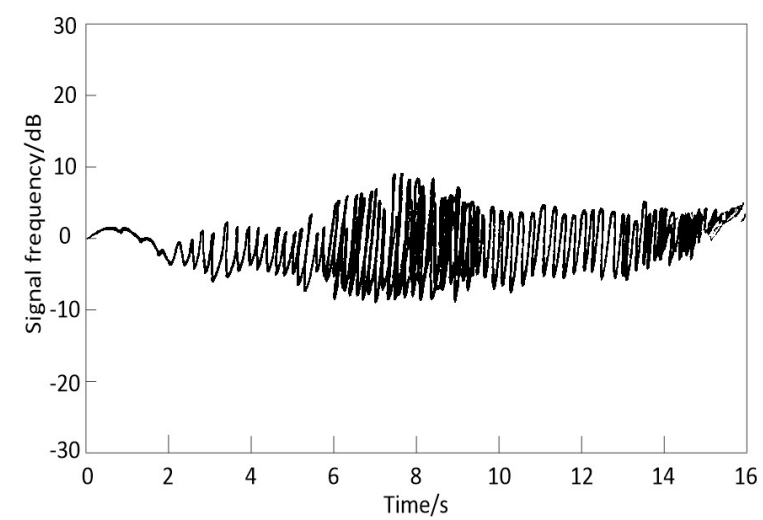

(b) Denoising results of face recognition method based on GABP neural network algorithm

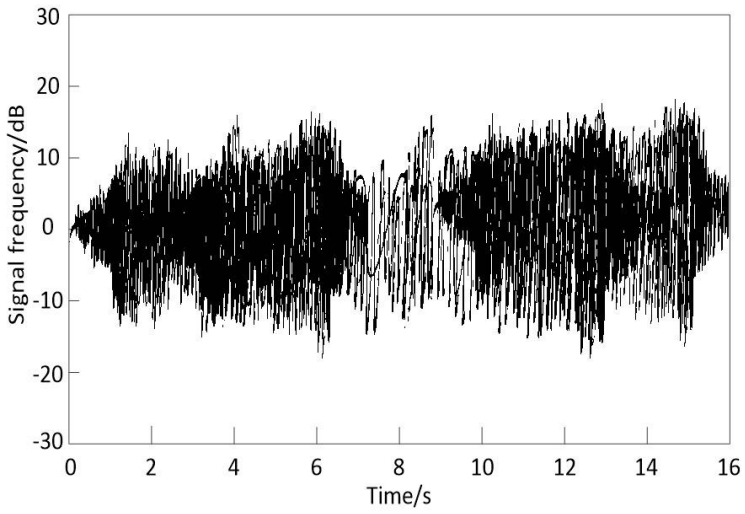

(d) Denoising results of feature weighted fusion face recognition method based on deep neural network

Figure 3: Denoising results of three different methods

ture and feature weighted fusion face recognition method based on deep neural network. By comparing Figure 3(c), 3(d) and 3(a), it is found that the signal in the face image after the denoising with two methods has a large fluctuation, which is very different from the signal frequency in the original face image. Compared with the denoising results of three different methods, it is found that the face recognition method based on GA-BP neural network algorithm can effectively remove the noise signal in the face image. Because in the face recognition method based on GA-BP neural network algorithm, an image sparse representation method based on dictionary is used to construct mixed denoising model to remove salt and pepper noise, impact noise and Gauss noise in the face image. The denoising effect is good, and the signal to noise ratio is high.

$\varsigma$ is the sensitive factor. If the value of the sensitive factor is taken within the interval $[0,10]$, the greater the value of the sensitive factor is, the more accurate the feature vector is, and the higher the accuracy of face recognition is. The face recognition method based on GA-BP neural network algorithm, the 3D face recognition method based on the neighborhood structure of singular point and the feature weighted fusion face recognition method based on the depth neural network are tested by the sensitive factor, and the accuracy rate of the face recognition by the three different methods is compared. The test results are shown in Figure 4.

Figure 4(a) is a test result of face recognition method based on GA-BP neural network algorithm. Analysis of Figure 4(a) shows that the sensitivity factor obtained by this method is more than 7 in many iterations. Figure 4(b) is a test result of 3D face recognition method based on singular point neighborhood structure. Figure 4(c) is the result of feature weighted fusion face recognition method 


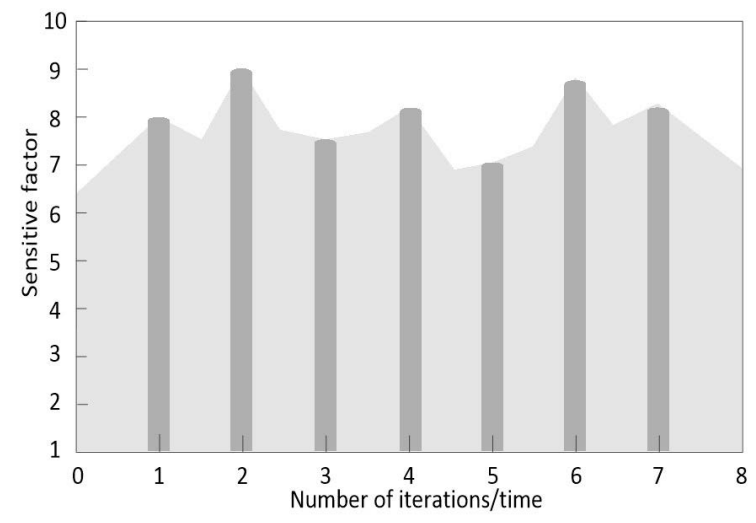

(a) The signal frequency of the original face image

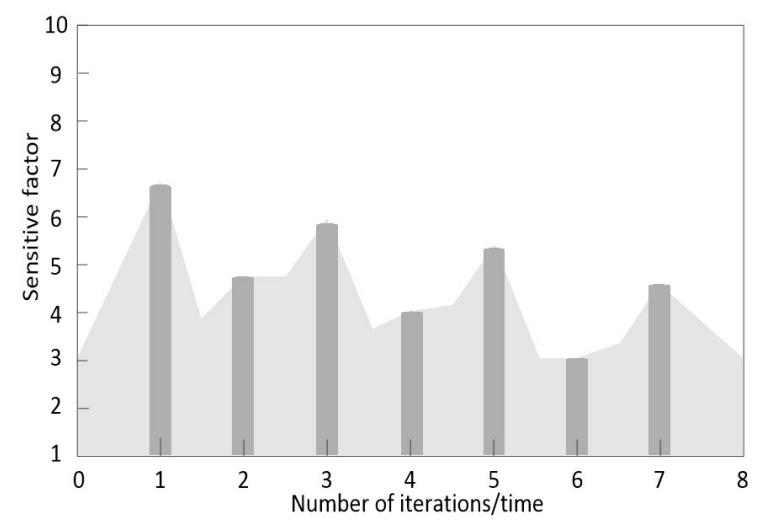

(b) Test results of face recognition method based on GA-BP neural network algorithm

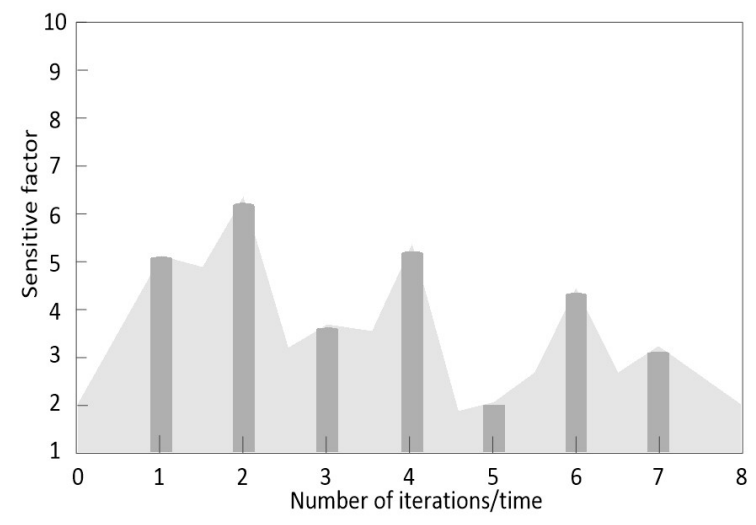

(c) Test results of feature weighted fusion face recognition method based on deep neural network

Figure 4: Test results of three different methods

based on deep neural network. Analysis of Figures 4(b) and 4(c) show that the sensitivity factors obtained by the above two methods in multiple iterations are as low as 3 and 2, respectively. Comparing the test results of three different methods, it can be seen that the sensitivity factor of face recognition method based on GA-BP neural network algorithm is higher. The bigger the value of the sensitive factor is, the more accurate the eigenvector is. The recognition accuracy of face recognition method based on GA-BP neural network algorithm is verified.

\section{Discussion}

According to the above analysis, the face recognition method based on a GA-BP neural network algorithm can remove the salt and pepper noise, the impact noise and Gauss noise in the face image, and complete the face recognition accurately. Recognition efficiency is also an important factor in face recognition. The face recognition method based on GA-BP neural network algorithm, the 3D face recognition method based on the neighborhood structure of singular point and the face recognition method based on the feature weighted fusion based on the depth neural network are tested. Three different methods used to calculate sensitive factors in Experiment 2 are compared, and the results are shown in Figure 5.

Figure 5(a) is the time used in face image recognition based on GA-BP neural network algorithm. Analysis Figure 5(a) shows that the time of face image recognition is below $10 \mathrm{~s}$ when using this method. Figure 5(b) and 5(c) are the time used in face image recognition by using the $3 \mathrm{D}$ face recognition method based on singular point neighborhood structure and the feature weighted fusion face recognition method based on deep neural network. Analysis of Figures 5(b) and 5(c) shows that the recognition time of the above two methods is as high as $15 \mathrm{~s}$ and $18 \mathrm{~s}$, respectively. Compared with the test results of three different methods, the face recognition method based on GA-BP neural network algorithm uses less recognition time. Because the face recognition method based on GA-BP neural network algorithm realizes the K-L transformation of the sample space by solving the matrix eigenvalues and eigenvectors with the small dimension, which reduces the computational complexity of the algorithm and improves the recognition efficiency of the method. It is verified that face recognition method based on a GA-BP neural network algorithm has high recognition efficiency.

In summary, the face recognition method based on a GA-BP neural network algorithm has high signal-to-noise ratio, high recognition accuracy and high recognition efficiency. 


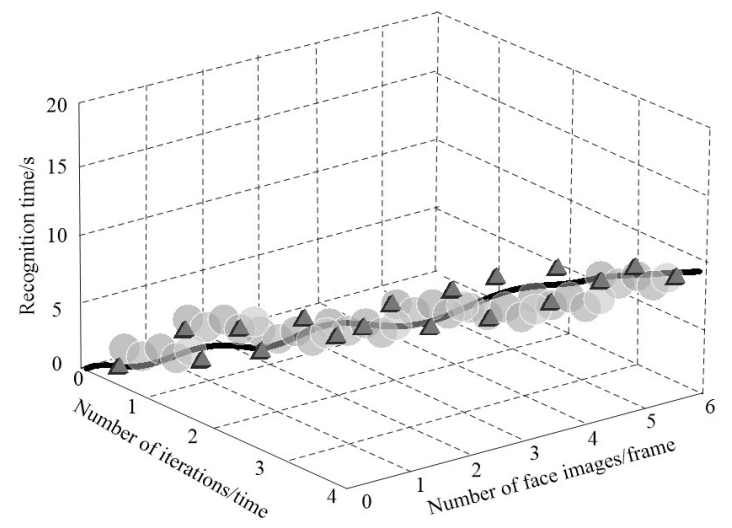

(a) Recognition time of face recognition method based on GABP neural network algorithm

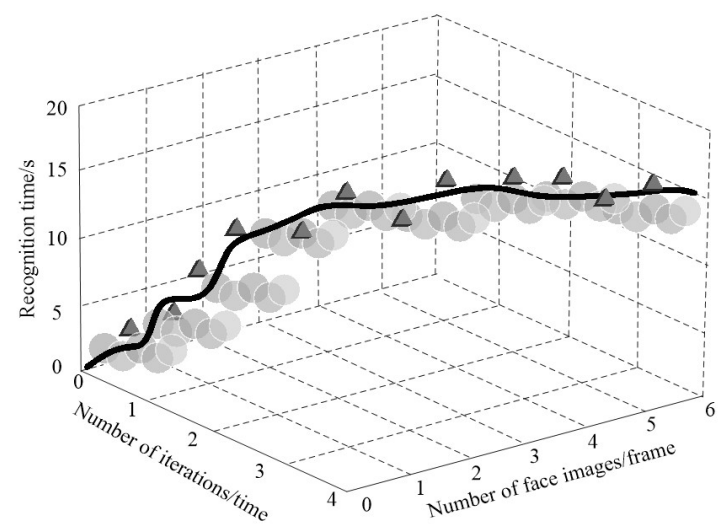

(b) Recognition time of feature weighted fusion face recognition method based on deep neural network

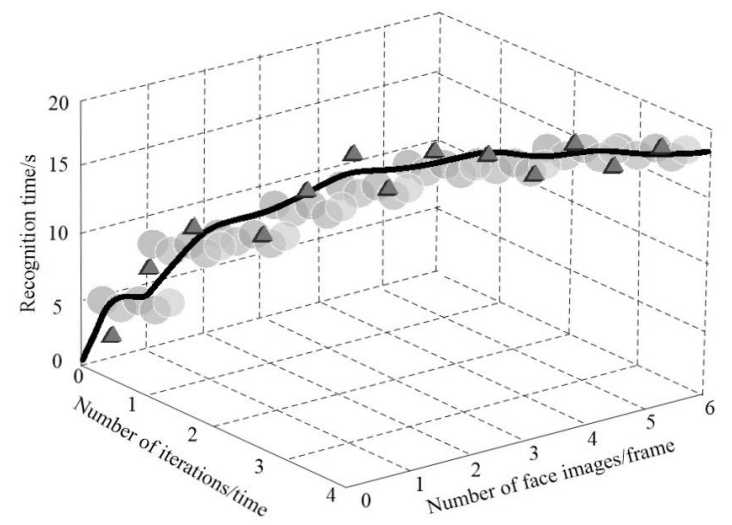

(c) Recognition time of feature weighted fusion face recognition method based on deep neural network

Figure 5: Recognition time of three different methods

\section{Conclusions}

As a new biometric recognition technology, face recognition technology has the advantages of friendliness, conve- nience and stability, and has attracted wide attention. This technology has broad market prospects and huge market value. At present, face recognition algorithm has the problems of low signal to noise ratio, low recognition accuracy and low recognition efficiency. A face recognition method based on a GA-BP neural network algorithm is proposed. The first step is to construct a mixed denoising model; the second step is to extract the feature vectors of face images; the third step is to use GA-BP neural network algorithm to complete face recognition.

Face recognition is an interdisciplinary and challenging problem. At present, most of the methods still remain in the research stage, the various theories still need to be further improved, and the recognition effect is still a great distance from the practical. Face recognition system is relatively complex and involves a lot of contents. Later work is mainly in the following aspects:

Face recognition method based on a GA-BP neural network algorithm has certain improvement on the recognition effect of face image. But there is still a certain gap between the actual application and the next work is to create a new database to conduct actual tests.

The sample of face image has a great influence on the result of recognition. The more samples there are, the more convenient the learning process is. However, for some special cases, if the sample is very limited, the possibility of misjudging in the unknown change recognition process of some face images is increased. How to effectively expand the existing samples is a development direction worth studying.

In the face recognition method based on GA-BP neural network algorithm, the feature extraction method of principal component analysis is used to complete the feature extraction of face image. If it can combine other feature extraction methods, it can get better results by integrating different features.

Because the face recognition process is an ill conditioned learning process from three dimensions to two dimensions, there is a certain error. In order to identify face images more accurately, direct 3D face recognition is an effective research method.

As a means of authentication, single biometric identification technique is difficult to ensure its stability and reliability. Therefore, the fusion of multi-feature biometrics is a promising research direction.

\section{References}

[1] Feng J.Y., Chen H.Y., Research on Face Recognition Based on Artificial Neural Network, Automat. Instrument., 2017, (5), 24-26. 
[2] Xu Y.J., Li W.X., Research on Face Recognition Based on the Gabor Wavelet and the Neural Network, J. China Acad. Electr. Inform. Technol., 2017, 12 (5), 534-539.

[3] Wang R., Based on the Research of Adaboost Face Recognition Algorithm, Electr. Design Eng., 2017, 25 (16), 190-193.

[4] Long H.Q., Tan T.Z., Based on the Depth of The convolution Network and Local Binary Pattern of Face Recognition, Comp. Simul., 2017, 34 (1), 322-325.

[5] Liu L.L., Research on Image Segmentation Technology for a License Plate Recognition, Bulletin Sci. Technol., 2017, 33(4), 125129.

[6] Yuan H., Wang Z.H., Jiang W.T., 3D Face Recognition Approach based on Singular Point neighborhood structure, Contr. Decis., 2017, 32 (10), 1739-1748.

[7] Sun J.G., Meng F.Y., Face Recognition based on Deep Neural Network and Weighted Fusion of Face Features, J. Comp. Appl., 2016, 36 (2), 437-443.

[8] Li Y.Q., Zhang S.W., Li H.B., Face Recognition Method Using Gabor Wavelet and Cross-covariance Dimensionality Reduction, J. Electr. Inform. Technol., 2017, 39 (8), 2023-2027.

[9] Lin Y.F., Zhang L.H., A Face Recognition Method Using Greedy Approximation Algorithm, Contr. Eng. China, 2017, 24 (10), 21252129.

[10] Gu G., Hou Z., Chen C., A Dimensionality Reduction Method based on Structured Sparse Representation for Face Recognition, Artif. Intel. Rev., 2016, 46 (4), 1-13.

[11] Liu L., Fieguth P., Zhao G., Extended Local Binary Patterns for Face Recognition, Inform. Sci., 2016, 358-359 (C), 56-72.

[12] Ji H.K., Sun Q.S., Ji Z.X., Collaborative Probabilistic Labels for Face Recognition from Single Sample per Person, Pattern Recog., 2017, 62 (C), 125-134.

[13] Wen Y., Zhang L., Deneen K.M.V., Face Recognition Using Discriminative Locality Preserving Vectors, Digit. Sign. Proc., 2016, 50 (C), 103-113.

[14] Kim Y.H., Kim H., Kim S.W., Illumination Normalisation Using Convolutional Neural Network with Application to Face Recognition, Electr. Let., 2017, 53 (6), 399-401.
[15] Rajeshwari J., Karibasappa K., Adaboost Modular Tensor Locality Preservative Projection: Face Detection in Video Using Adaboost Modular-based Tensor Locality Preservative Projections, LET Comp. Vis., 2017, 10 (7), 670-678.

[16] Xu W., Shen Y., Bergmann N., Sensor-assisted Multi-view Face Recognition System on Smart Glass, IEEE Trans. Mobile Comp., 2018, PP (99), 1-1.

[17] Kong Y.H., Wang Z.H., Che L.L., Real-time Face Recognition in Videos based on Convolutional Neural Networks (CNN) and CUDA, Sci. Technol. Eng., 2016, 16 (35), 96-100.

[18] Lu J.L., Chen J., Yang Z., Cloud Computing-based Embedded Face Recognition System Construction and Research, Comp. Meas. Contr., 2016, 124 (4), 146-148.

[19] Liu S., Zhang X., Peng Y., Virtual Images Inspired Consolidate Collaborative Representation-based Classification Method for Face Recognition, J. Mod. Opt., 2016, 63 (12), 1-8.

[20] Turhan C.G., Bilge H.S., Class-wise Two-dimensional PCA Method for Face Recognition, LET Comp. Vis., 2017, 11 (4), 286 300.

[21] Gasparic H.V., Drmic Z., Cacija M., Grasa Z., Petrak I., Bazok R. et al., Impact of Environmental Conditions and Agro-Technical Factors On Ground Beetle Populations in Arable Crops, Appl. Ecol. Envir. Res., 2017, 15(3), 697-711.

[22] Birs I., Muresan C., Folea S., Prodan O., A Comparison Between Integer and Fractional Order Pd $\mu$ Controllers for Vibration Suppression, Appl. Math. Nonlin. Sci., 2016, 1(1), 273-282.

[23] Gao W., Wang W., New Isolated Toughness Condition for Fractional (G, F, N) - Critical Graph, Colloq. Math., 2017, 147(1), 5565.

[24] Jiang S.C., Ge S.B., Wu X., Yang Y.M., Chen J.T., Peng W.X., Treating N-Butane by Activated Carbon and Metal Oxides, Toxicol. Envir. Chem., 2017, 99(5-6), 753-759.

[25] Sudhakar S., Francis S., Balaji V., Odd Mean Labeling for Two Star Graph, Appl. Math. Nonlin. Sci., 2017, 2(1), 195-200.

[26] Pablo-Romero M.D.P., Pozo-Barajas R., Sanchez-Braza A., Analyzing the Effects of the Benchmark Local Initiatives of Covenant of Mayors Signatories, J. Clean. Prod., 2018, 176, 159-174. 